\title{
MID-INFRARED SPECTRAL DIAGNOSTICS OF LUMINOUS INFRARED GALAXIES
}

\author{
A. O. Petric ${ }^{1}$, L. Armus ${ }^{1}$, J. Howell ${ }^{1}$, B. Chan $^{2}$, J. M. Mazzarella ${ }^{2}$, A. S. Evans ${ }^{3,4}$, J. A. Surace ${ }^{1}$, D. Sanders ${ }^{5}$,

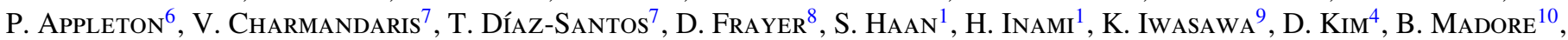 \\ J. Marshall ${ }^{1}$, H. Spoon ${ }^{11}$, S. Stierwalt ${ }^{1}$, E. Sturm ${ }^{12}$, V. U ${ }^{4,15,16}$, T. VAVILKin ${ }^{13}$, And S. VeILleuX ${ }^{14}$ \\ ${ }^{1}$ Spitzer Science Center, California Institute of Technology, 1200 E. California Blvd., Pasadena, CA 91125, USA \\ ${ }^{2}$ Infrared Processing Center, California Institute of Technology, 770 S. Wilson Ave., Pasadena, CA 91125, USA \\ ${ }^{3}$ Department of Astronomy, University of Virginia, P.O. Box 400325, University of Virginia, Charlottesville, VA 22904, USA \\ ${ }^{4}$ National Radio Astronomy Observatory, 520 Edgemont Road, Charlottesville, VA 22903-2475, USA \\ ${ }^{5}$ Institute for Astronomy, University of Hawaii, 2680 Woodlawn Drive, Honolulu, HI 96822, USA \\ ${ }^{6}$ NASA Herschel Science Center, 770 S. Wilson Ave., Pasadena, CA 91125, USA \\ ${ }^{7}$ University of Crete, Department of Physics, GR 71003, Heraklion, Greece \\ ${ }^{8}$ National Radio Astronomy Observatory, P.O. Box 2, Green Bank, WV 24944, USA \\ ${ }^{9}$ ICREA and Institut de Ciencies del Cosmos (ICC), Universitat de Barcelona (IEEC-UB), Marti i Franques, 1, 08028 Barcelona, Spain \\ ${ }^{10}$ Carnegie Observatories, 813 Santa Barbara Street, Pasadena, CA 91101, USA \\ 11224 Space Sciences Building, Cornell University, Ithaca, NY 14853, USA \\ ${ }^{12}$ MPE, Postfach 1312, 85741 Garching, Germany \\ ${ }^{13}$ Department of Physics and Astronomy, State University of New York at Stony Brook, NY 11794, USA \\ ${ }^{14}$ Department of Astronomy, University of Maryland, College Park, MD 20742, USA \\ Received 2010 September 8; accepted 2010 December 8; published 2011 March 1
}

\begin{abstract}
We present a statistical analysis of the mid-infrared (MIR) spectra of 248 luminous infrared (IR) galaxies (LIRGs) which comprise the Great Observatories All-sky LIRG Survey (GOALS) observed with the Infrared Spectrograph (IRS) on board the Spitzer Space Telescope. The GOALS sample enables a direct measurement of the relative contributions of star formation and active galactic nuclei (AGNs) to the total IR emission from a large sample of local LIRGs. The AGN contribution to the MIR emission $\left(f_{\mathrm{AGN}}\right)$ is estimated by employing several diagnostics based on the properties of the [Ne v], [O IV], and [Ne II] fine-structure gas emission lines, the $6.2 \mu \mathrm{m}$ polycyclic aromatic hydrocarbon (PAH), and the shape of the MIR continuum. We find that $18 \%$ of all LIRGs contain an AGN and that in $10 \%$ of all sources the AGN contributes more than $50 \%$ of the total IR luminosity. Summing up the total IR luminosity contributed by AGNs in all our sources suggests that AGNs supply $\sim 12 \%$ of the total energy emitted by LIRGs. The average spectrum of sources with an AGN looks similar to the average spectrum of sources without an AGN, but it has lower PAH emission and a flatter MIR continuum. AGN-dominated LIRGs have higher IR luminosities, warmer MIR colors, and are found in interacting systems more often than pure starburst LIRGs. However, we find no linear correlations between these properties and $f_{\mathrm{AGN}}$. We used the IRAC colors of LIRGs to confirm that finding AGNs on the basis of their MIR colors may miss $40 \%$ of AGN-dominated (U)LIRGs.
\end{abstract}

Key words: galaxies: evolution - galaxies: interactions

Online-only material: color figures

\section{INTRODUCTION}

The Infrared Astronomical Satellite (IRAS) provided the first unbiased survey of the sky at mid-infrared (MIR) and farinfrared (FIR) wavelengths, giving us a comprehensive census of the infrared (IR) emission properties of galaxies in the local universe. IR number counts have been used to trace the importance of IR emission as a function of redshift, to explore star formation and galaxy evolution (Flores et al. 1999; Gispert et al. 2000; Franceschini et al. 2001; Chary \& Elbaz 2001; Chary et al. 2004; Elbaz et al. 2002; Lagache et al. 2003; Marleau et al. 2004; Le Floc'h et al. 2005; Caputi et al. 2006; Magnelli et al. 2009).

To understand the origin of the observed IR emission, MIR diagnostic tools based on the Infrared Space Observatory (for an exhaustive review, see Genzel \& Cesarsky 2000) have been developed to study the roles played by star formation,

\footnotetext{
${ }^{15}$ NASA Jenkins Predoctoral Fellow.

${ }^{16}$ SAO Predoctoral Fellow, Harvard-Smithsonian Center for Astrophysics, Cambridge, MA 02138, USA.
}

active galactic nucleus (AGN), and shocks (interaction driven and wind driven) in producing the observed IR emission. These diagnostics permit a direct mapping between IR number counts as a function of redshift/luminosity/galaxy type and the evolution of accretion and star formation.

The most basic diagnostics employed to estimate the AGN contribution to the MIR emission in individual galaxies are the ratios of high- to low-ionization fine-structure emission lines: [Ne v] $14.3 \mu \mathrm{m} /[\mathrm{Ne}$ II] $12.8 \mu \mathrm{m}$ and [O IV] $25.9 \mu \mathrm{m} /[\mathrm{Ne}$ II] $12.8 \mu \mathrm{m}$. The [Ne v] $14.3 \mu \mathrm{m}$ and [O IV] $25.9 \mu \mathrm{m}$ lines trace high-ionization gas. These methods have been used by, e.g., Lutz et al. (1999), Genzel et al. (1998), Verma et al. (2003), Armus et al. (2006), Sturm et al. (2002), Spoon et al. (2007), Armus et al. (2007), and Farrah et al. (2007). The ionization potential of [Ne v] is $96 \mathrm{eV}$. This is too high to be produced by OB stars, therefore its detection in the integrated spectrum of a galaxy usually indicates the presence of an AGN. This is not true for the [O IV] line because it takes only $55 \mathrm{eV}$ to ionize $\mathrm{O}^{++}$. Empirically it has been shown that emission line ratios of $[\mathrm{Ne} \mathrm{V}] /[\mathrm{Ne}$ II $] \geqslant 0.75$ and $\left[\mathrm{O}_{\mathrm{IV}}\right] /[\mathrm{Ne} \mathrm{II}] \geqslant 1.75$ indicate that more than $50 \%$ of the nuclear MIR emission is 
produced by an AGN (e.g., Armus et al. 2007 and references therein).

Additional diagnostics of the relative contribution of starbursts (SBs) and AGNs to the MIR luminosity are based on the dust properties, in particular polycyclic aromatic hydrocarbon (PAH) emission lines and the continuum emission arising from dust being heated by an SB or an AGN. Mid-infrared continuum emission in galaxies arises from a combination of ionized interstellar gas, evolved stellar population, non-thermal emission from radio sources, very small grains, and PAHs. Empirically their respective contributions can be roughly distinguished from the shape of the spectral energy distribution (SED; Laurent et al. 2000). Both theoretical models (e.g., Pier \& Krolik 1992; Nenkova et al. 2002; Granato et al. 1997; Levenson et al. 2007) and observations (e.g., Alonso-Herrero et al. 2001) show that the AGN radiation field can heat grains such that the dust continuum emission becomes prominent between 3 and $6 \mu \mathrm{m}$. Galaxies with an AGN tend to have low $6.2 \mu \mathrm{m}$ PAH equivalent width (EQW; e.g., Genzel et al. 1998; Lutz et al. 1999; Rigopoulou et al. 1999; Tran et al. 2001; Sturm et al. 2000; Desai et al. 2007; Wu et al. 2009) due to the presence of a significant hot dust continuum and also because the hard AGN photons may destroy the PAH molecules.

Spitzer IRS observations of normal nearby optically classified galaxies confirmed that the MIR diagnostics described above, diagnostics based on a combination of high- to low-ionization line ratios, and $\mathrm{PAH}$ strengths are indeed very effective at determining the AGN versus SB contribution to the IR in nearby galaxies. These studies such as the Spitzer Infrared Nearby Galaxy Survey were based on galaxies from a wide range of environments, Hubble types, and dust contents (Dale et al. 2006). Most recently Goulding \& Alexander (2009) looked at an optically selected nearby $(D<15 \mathrm{Mpc}$ ) sample of galaxies with IR luminosity between $3 \times 10^{9} L_{\odot}$ and $2 \times 10^{11} L_{\odot}$ and found that $27 \%$ have AGNs based on [Ne v] detections. Excluding the nearby luminous infrared galaxy (LIRG) NGC1068, the [Ne v]/ [Ne II] ratios and the PAH EQWs of these sources suggest that only $5 \%$ of sources are AGN-dominated. IRS studies of 24 nearby SBs with luminosities between $10^{9.75}$ and $10^{11.6}$ at an average distance of $33 \mathrm{Mpc}$ provided high- and lowresolution spectral templates for pure SBs (Bernard-Salas et al. 2009; Brandl et al. 2006). Brandl et al. (2006) also found that the spectral continuum slope longward of $15 \mu \mathrm{m}$ can be used to discriminate between SB and AGN-powered sources. These authors also found that in pure SBs the PAH EQWs are independent of $L_{\mathrm{IR}}$ but that the luminosity of the PAH feature scales with $L_{\mathrm{IR}}$, in particular the $6.2 \mu \mathrm{m}$ feature can be used to approximate the total IR of the SB.

Ultraluminous infra-red galaxies (ULIRGs; $L_{\mathrm{IR}[8-1000 \mu \mathrm{m}]} \geqslant$ $10^{12} L_{\odot}$ ) have also been extensively studied in the MIR regime. ULIRGs were chosen primarily from the $1 \mathrm{Jy}$ survey (Kim et al. 1998), the IRAS 2 Jy survey (Strauss et al. 1992), and the FIRST/IRAS radio-far-infrared sample of Stanford et al. (2000) with redshifts between 0.018 and 0.93 , and IR luminosities in the range $10^{11.7}-10^{13.3} L_{\odot}$ were observed with the IRS by Armus et al. (2004, 2006, 2007), Desai et al. (2007), and Farrah et al. (2007). These authors determine that around $42 \%$ of ULIRGs contain an AGN as indicated by the detection of the [Ne v] emission line, that in $20 \%-40 \%$ of ULIRGs the AGN dominates the MIR emission, and that the $6.2 \mu \mathrm{m}$ PAH EQW is anti-correlated with the $24 \mu \mathrm{m}$ luminosity and with the IRAS $25 / 60 \mu \mathrm{m}$ color. More recently Veilleux et al. (2009a) presented IRS observations of 74 ULIRGs and 34 Palomar-
Green (PG) quasars (QSOs). These authors derived adjustments to the MIR diagnostics to estimate the AGN contribution to the total bolometric luminosity not just to the MIR luminosity. They estimated that the average AGN contribution to the bolometric luminosity of ULIRGs is on average $35 \%-40 \%$ and that it ranges from $15 \%-35 \%$ among cool optically classified H IIlike and LINER ULIRGs to 50\%-75\% among warm Seyfert 2 and Seyfert 1 ULIRGs.

While nearby normal and SB galaxies as well as ULIRGs have been studied in detail with IRS, MIR spectroscopic studies have only recently unveiled the source of IR emission in LIRGs. LIRGs $\left(L_{\mathrm{IR}[8-1000 \mu \mathrm{m}]} \geqslant 10^{11} L_{\odot}\right.$, with $L_{\mathrm{IR}}$ as defined in Sanders $\&$ Mirabel 1996) emit the bulk of their energy in the far-infrared. LIRGs account for $\geqslant 50 \%$ of the total $24 \mu \mathrm{m}$ galaxy population with $f_{24 \mu \mathrm{m}} \geqslant 80 \mathrm{Jy}$ and for $\sim 50 \%$ of the comoving star formation density at $z \sim 1$ (e.g., Le Floc'h et al. 2005; Caputi et al. 2006; Magnelli et al. 2009). Unlike ULIRGs, LIRGs span the full range of galaxy interactions from non-merging spirals to late stage mergers, bridging the luminosity gap between SBs and QSOs.

Alonso-Herrero et al. (2010) and Pereira-Santaella et al. (2010) combine optical and MIR spectroscopy to determine the excitation conditions in a sample of 15 local LIRGS with IR luminosities $L_{\mathrm{IR}}=10^{11.5}-10^{11.59} L_{\odot}$.

These authors find that most of the LIRGs have MIR spectroscopic properties similar to pure SBs and that the integrated values show, in general, larger $6.2 \mu \mathrm{m}$ PAH EQW than the nuclear spectra suggesting that star formation partially masks the nuclear activity.

However, until recently there has been no comprehensive study of the MIR spectra of a large sample of LIRGs. This paper employs that MIR diagnostics aimed at isolating the AGN contribution to the IR emission in the study of 248 LIRGs. The Great Observatories All-sky LIRG Survey (GOALS) targets a complete sample of 202 systems in the local universe selected from the IRAS Revised Bright Galaxy Sample (RBGS; Sanders et al. 2003). GOALS brings together Hubble Space Telescope (HST; UV, optical, and NIR), Spitzer (imaging and spectroscopy), Galaxy Evolution Explorer, Chandra, and NIR ground-based spectroscopic data to understand low $-z(\leqslant 0.088)$ LIRGs. The broad wavelength coverage of this study can be used to trace the young and old stars, the dust, the hot ionized gas, and the warm molecular gas in LIRGs, making this data set unmatched in its power to shed light on the genesis of SBs and the growth of black holes in interacting galaxies locally. An outline of the GOALS project and a multi-wavelength analysis of the LIRG VV340 are given in Armus et al. (2009). Evans et al. (2008) and Inami et al. (2010) present a similar multi-wavelength analysis of LIRGs NGC 2623 and CGCG448020, respectively. Howell et al. (2010) compares the IR and UV properties of LIRGs and Díaz-Santos et al. (2010) discusses the extent of the MIR emission across a large sample of LIRGs.

A key component of GOALS is the mid-infrared spectroscopic study of 248 LIRG nuclei (in 202 systems) with the Infrared Spectrograph (IRS) on Spitzer. In the present paper, we discuss the first statistical results from GOALS on the MIR nuclear spectra focusing on the ionization source in LIRG nuclei at low redshift. In Section 2, we describe the IRS observations and analysis. In Section 3, we present the results based on key SB and AGN emission line features that are used to diagnose the origin of the IR emission as well as statistics on the presence and strength of these features in the nuclei of LIRGs. In 
Section 3, we also present an analysis of the properties of average low-resolution spectra obtained from samples of LIRGs without and with an AGN. In Sections 4.1-4.3, we present an investigation of how these diagnostics relate to the global properties of LIRGs. In Section 4.4, we discuss the connections between AGNs and merger activity in LIRGs.

In Section 4.5, we compare spectroscopic diagnostics of AGNs to diagnostics using only IR photometry. In the conclusion we summarize the findings of this work. This is the first in a series of papers which compare the MIR spectral and multi-wavelength properties of LIRGs in the local universe.

\section{IRS OBSERVATIONS AND DATA ANALYSIS}

Spectra of 248 individual nuclei in 202 LIRG systems, observed in all four IRS modules (Short-Low, Long-Low, Short-High, and Long-High), were used for this investigation. The widths of the SL, SH, LL, LH slits $\left(3^{\prime \prime} .6,44^{\prime \prime} 7,10^{\prime \prime} 7,11^{\prime \prime} .1\right)$ correspond to $1.5,2.0,4.5,4.6 \mathrm{kpc}$, respectively, at a distance of $88 \mathrm{Mpc}$ (the median galaxy distance of our sample). As part of GOALS, IRS spectra for 158 LIRG systems were obtained (PID 30323; Armus et al. 2009). Of these, 115 were observed in all four IRS modules, while 43 have been observed in three or fewer IRS modules in order to complete the existing archival data and to ensure complete coverage for all GOALS targets. Spectra for the remaining 44 LIRG systems were obtained from other Spitzer IRS programs in the archive. In all data from PID 30323, IRS Staring Mode was employed, using "cluster target" observations for those sources with well-separated $\left(\Delta r \geqslant 10^{\prime \prime}\right)$, nearby interacting companions. Among the 202 LIRGs studied, secondary nuclei were targeted only when the flux ratio of primary to secondary nucleus (as measured in the MIPS $24 \mu \mathrm{m}$ data) is less than or equal to five, in order to capture the spectra of the nuclei actively participating in the far-infrared emission of the system. The IRS data on two LIRGs (IIIZw35 and NGC 1614) in the GOALS sample do not include spectra of the nuclear regions, and as such they are not included in the statistics presented below.

All data were reduced using the S15, S16, and S17 IRS pipelines at the Spitzer Science Center. ${ }^{17}$ The pipelines were changed to modify certain header keywords, produce new flat fields, and lower SL and LL fringes by $1 \%-20 \%$, provide better treatment of ramp slopes, and make several changes to the post-BCD products which we did not use for this analysis. The changes made between the pipelines should not systematically alter the measurements presented here. The IRS pipeline includes ramp fitting, dark sky subtraction, droop correction, linearity correction and wavelength, and flux calibration.

The backgrounds in the high-resolution data were subtracted for all objects with dedicated sky observations $(\sim 60 \%$ of the sources). For the low-resolution data, and for high-resolution data without dedicated background observations, off-source nods were used for sky subtraction. Large objects in PID 30323 had dedicated background pointings for both low- and highresolution data. Bad pixel mask files were combined such that the final masks flagged all the individual bad pixels, if they were marked as bad in one individual exposure. Basic calibrated data sets (BCDs) for each nod were combined by determining the median if more than five BCDs were available, otherwise the average was used with $3 \sigma$ clipping.

\footnotetext{
17 http://ssc.spitzer.caltech.edu/irs/features/
}

Each nod was extracted with SPICE $^{18}$ using the standard extraction aperture and point-source calibration. Nod 1 and nod 2 were compared with each other, and pixels were flagged if the difference between nod 1 and nod 2 exceeded $30 \%$ and when adjacent pixels within the same nod differed by more than $30 \%$ (due to a cosmic ray or hot pixel). Spatial profiles were computed from the SL data at 8.6, 10, and $12.8 \mu \mathrm{m}$ and discussed in detail in the GOALS IRS delivery documents. ${ }^{19}$ From these data it was assessed that $20 \%$ of the sources have extended emission at $12.8 \mu \mathrm{m}$ while the remaining $80 \%$ are point sources.

Twenty-eight systems were observed in spectral-mapping mode. The two-dimensional BCDs were assembled, then cleaned to remove obvious spikes, and finally the nuclear spectra were extracted with CUBISM (Smith et al. 2007) using extraction regions of sizes equal to those of extraction regions for point sources in the spectra taken in staring mode. In total 36 nuclear spectra were extracted from data taken in spectralmapping mode.

Gaussian fits were performed on the high-resolution data to measure the fluxes for each of the gas emission lines and to deblend the [Cl II] $14.368 \mu \mathrm{m}-[\mathrm{Ne} \mathrm{V}] 14.322 \mu \mathrm{m}$ lines and the [O IV] $25.890 \mu \mathrm{m}-[\mathrm{Fe}$ II] $25.988 \mu \mathrm{m}$ lines. To properly estimate the $6.2 \mu \mathrm{m}$ PAH EQW, the associated continuum was measured by using a spline fit to the continuum but excluding points affected by water ice absorption between 5.55 and $6.0 \mu \mathrm{m}$ and hydrocarbon absorption. The amount of water ice and hydrocarbon absorption was estimated as described in Spoon et al. (2007). All the spectra were inspected by eye to determine which sources had water ice absorption. For those sources with water ice absorption (4\% of all spectra) the continuum was estimated by using a linear interpolation of all data points in the following wavelength regions: [5.1 $\mu \mathrm{m}-5.6 \mu \mathrm{m}]$, [6.7 $\mu \mathrm{m}-6.9 \mu \mathrm{m}]$, and [7.1 $\mu \mathrm{m}-7.2 \mu \mathrm{m}]$.

For each spectrum the $5.5 \mu \mathrm{m}$ flux was measured by integrating the continuum between 5.3 and $5.8 \mu \mathrm{m}$, and the $15 \mu \mathrm{m}$ flux was estimated by integrating continuum between 14 and $15 \mu \mathrm{m}$. The nuclear $24 \mu \mathrm{m}$ flux was calculated from the IRS LL spectrum using the spectral response of the MIPS $24 \mu \mathrm{m}$ bandpass. The total 24 and $70 \mu \mathrm{m}$ fluxes were measured from MIPS observations (J. M. Mazzarella et al. 2011, in preparation) using typical apertures of 1 arcmin.

\section{RESULTS}

The high-resolution ( $\mathrm{SH}$ and LH) LIRG spectra are dominated by atomic fine-structure lines of $\mathrm{Ne}, \mathrm{O}, \mathrm{Si}, \mathrm{S}$ as well as warm $\mathrm{H}_{2}$. Several methods to determine the relative contribution of star formation versus AGN to the IR luminosity of LIRGs using MIR spectra are used. The results section is organized as follows: the ionized gas diagnostics of AGN activity based on high- and low-ionization fine-structure lines are discussed in Section 3.1; the results arising from the dust diagnostics are presented in Section 3.2; low-resolution spectra are divided into four groups based on the presence and importance of an AGN to the nuclear MIR emission as determined from the ionized gas diagnostics discussed in Section 3.1; the low-resolution spectra of each group of sources are then averaged and these average spectra are presented in Section 3.3. The properties of these average spectra and their implications for statistical studies of AGN activity are also briefly discussed in Section 3.3. All wavelengths mentioned here are rest-frame values.

\footnotetext{
18 http://ssc.spitzer.caltech.edu/dataanalysistools/tools/spice

19 http://irsa.ipac.caltech.edu/data/SPITZER/GOALS
} 
Table 1

[Ne v] $14.3 \mu \mathrm{m}$ Detections in the GOALS Sample

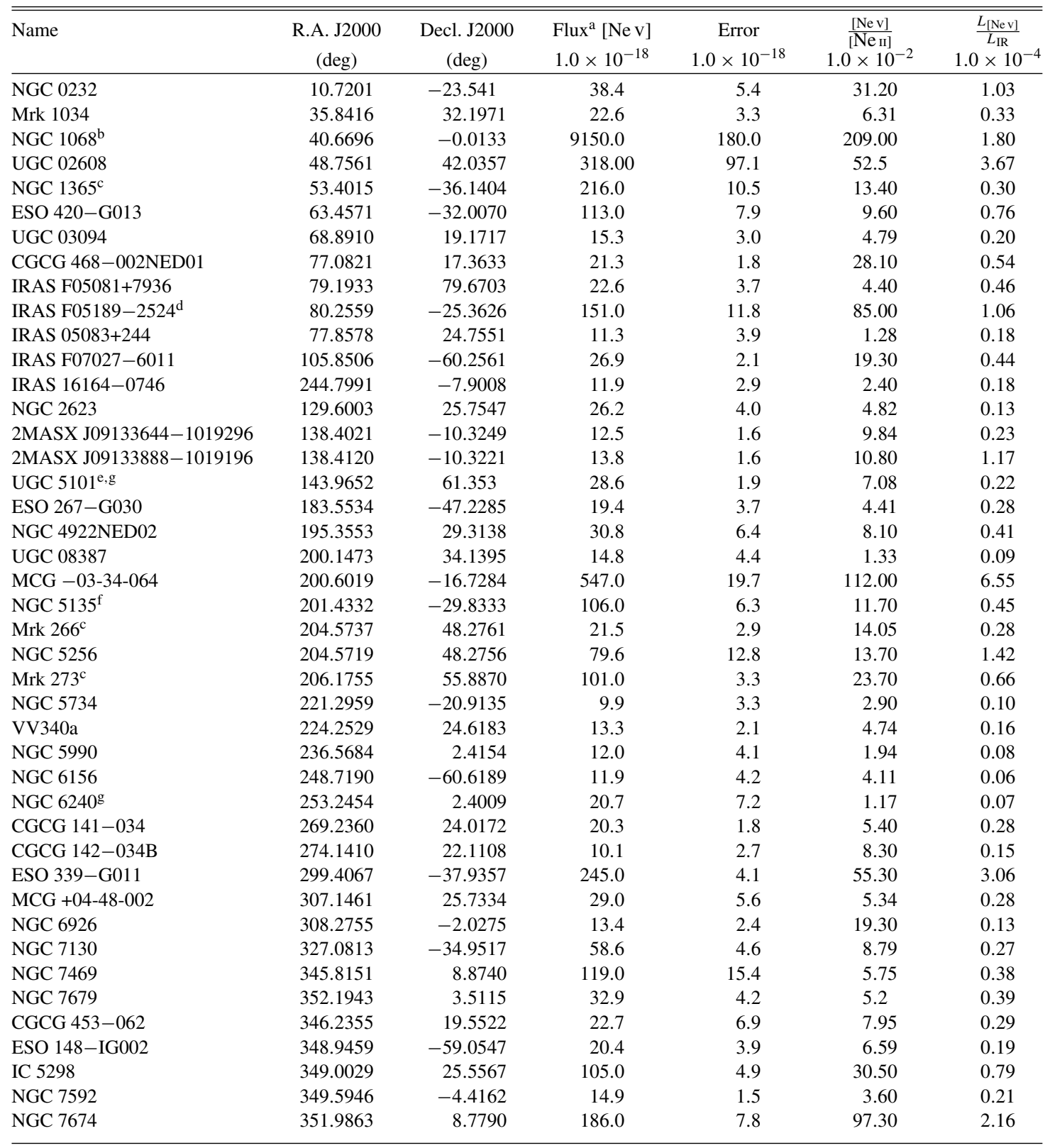

Notes. ${ }^{a}$ All fluxes are given in $\mathrm{W} \mathrm{m}^{-2}$. The fluxes presented here agree with previously published values within the errors, in all cases with the exception of fluxes for Mrk 266 and NGC5135.

${ }^{\mathrm{b}}$ Howell et al. (2007).

${ }^{\mathrm{c}}$ Dudik et al. (2007).

d Armus et al. (2007).

e Farrah et al. (2007).

${ }^{\mathrm{f}}$ Gorjian et al. (2007).

g Armus et al. (2006).

\subsection{Ionized Gas Diagnostics}

The [Ne v] $14.3 \mu \mathrm{m}$ line is detected in 43 nuclei representing $18 \%$ of the GOALS sample. Because detecting [Ne v] emission over kpc scales provides direct evidence for the presence of an AGN, the [Ne v] properties can be directly compared with other direct indicators of AGN activity (e.g., those based on X-ray emission; Iwasawa et al. 2011). Table 1 lists all the sources with [Ne v] detections at $14.3 \mu \mathrm{m}$ as well as their [Ne v]/[Ne II] ratios and the ratio $L_{[\mathrm{Nev}]} / L_{\mathrm{IR}}$. The detected [Ne v] fluxes range between $1.5 \times 10^{-18} \mathrm{~W} \mathrm{~m}^{-2}$ and $9 \times 10^{-15} \mathrm{~W} \mathrm{~m}^{-2}$ with a median flux of $2.27 \times 10^{-17} \mathrm{~W} \mathrm{~m}^{-2}$. The $[\mathrm{Nev}] /[\mathrm{Ne} \mathrm{II}]$ ratios range between $\sim 1 \times 10^{-3}$ and 2.09 , with a median of 0.07 . The $L_{\text {[Ne v] }} / L_{\text {IR }}$ range between $\sim 1 \times 10^{-5}$ and $\sim 1 \times 10^{-4}$. Note that the MIR spectra and [Ne v] fluxes of the ULIRGs in GOALS have already been published in Armus et al. (2004, 2006, 2007), Farrah et al. (2007), and Veilleux et al. (2009a).

Figures 1 and 2 show the ratios of $[\mathrm{Ne} \mathrm{V}] /[\mathrm{Ne}$ II] and $[\mathrm{O}$ IV] $/[\mathrm{Ne} I \mathrm{I}]$ versus the EQW of the $6.2 \mu \mathrm{m}$ PAH feature for the LIRGs in our sample. As mentioned in the introduction, 


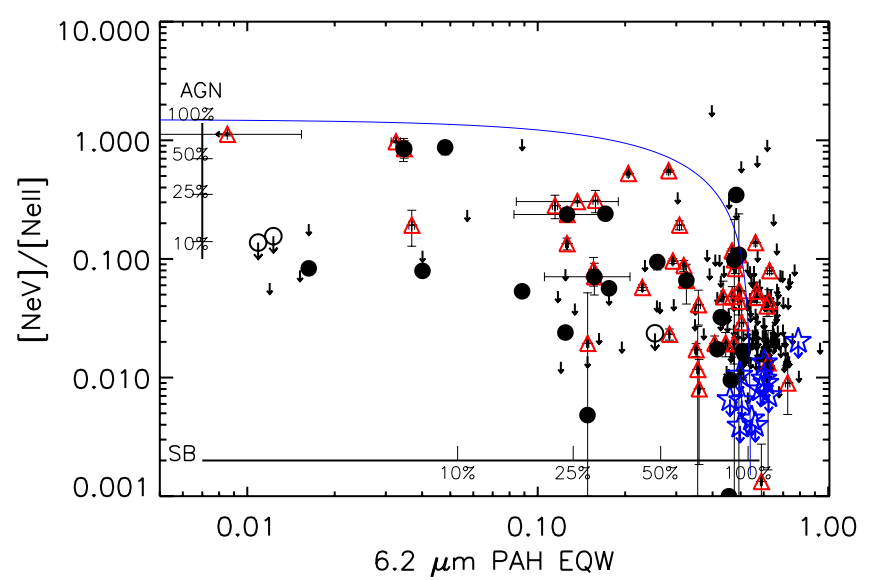

Figure 1. Mid-infrared [Ne v]/[Ne II] vs. $6.2 \mu \mathrm{m}$ PAH EQW excitation diagram. The red triangles are $[\mathrm{Ne} \mathrm{v}]$ detections while the black arrows are upper limits. In all cases $1 \sigma$ error bars are shown. The black circles indicate ULIRGs where the filled symbols show detections and empty symbols show upper limits. The blue empty stars mark upper limits for SB galaxies from Bernard-Salas et al. (2009). The solid black lines indicate the fractional AGNs and SB contribution to the MIR luminosity from the [Ne v]/[Ne II] (vertical) and $6.2 \mu \mathrm{m}$ PAH EQW (horizontal) assuming a simple linear mixing model. In each case, the $100 \%$, $50 \%, 25 \%$, and $10 \%$ levels are marked. The $100 \%$ level is set by the average detected values for the [Ne v]/Ne II] and $6.2 \mu \mathrm{m}$ EQW among AGNs and SBs, respectively, as discussed in Armus et al. (2007). The blue line traces where the summed SB and AGN contribution equals 100\%. For most LIRGs the [Ne v]/ [Ne II] ratio suggests that the AGN contribution to the nuclear MIR luminosity is below $10 \%$.

(A color version of this figure is available in the online journal.)

empirically it has been shown that emission line ratios of [ $\mathrm{Ne} \mathrm{v}] /$ $[\mathrm{Ne}$ II $] \geqslant 0.75$ and $[\mathrm{O}$ IV $] /[\mathrm{Ne} \mathrm{II}] \geqslant 1.75$ indicate that more than $50 \%$ of the nuclear MIR emission is produced by an AGN (e.g., Armus et al. 2007 and references therein). Three LIRG nuclei have $[\mathrm{Ne} \mathrm{v}] /[\mathrm{Ne} I \mathrm{II}] \geqslant 0.75$, implying that only $1 \%$ of LIRGs have more than $50 \%$ of their MIR emission powered by an AGN (see Figure 1).

For comparison with the LIRGs, nine SB galaxies are included in the figures using data from Bernard-Salas et al. (2009): NGC 660, NGC 1222, IC 342, NGC 1614, NGC 2146, NGC 3256, NGC 3310, NGC 4088, NGC 4676, NGC 4818, NGC 7252, and NGC 7714. Three of these galaxies are LIRGs while the rest have lower IR luminosities. These particular objects were used by Armus et al. (2007) to determine the zero points in the ionized gas diagnostics. Data for seven well-studied ULIRGs from the RBGS sample analyzed in Armus et al. (2004, 2007) are also presented for comparison: Mrk 231, Arp 220, IRAS 05189-2524, Mrk 273, IRAS 08572+3915, UGC 5101, and Mrk 1014.

The [O IV] $25.890 \mu \mathrm{m}$ line is detected in 120 nuclei $(53 \%$ of the sources). Four LIRGs have ratios of [O IV] $/[\mathrm{Ne}$ II $] \geqslant 1.75$ implying that only $1 \%$ of LIRGs have more than $50 \%$ of their MIR emission powered by an AGN. The ratios of [O IV]/ [Ne II] range between 0.002 and 5.5 and have a median of 0.03 and a mean of 0.24 with a dispersion of 0.74 (see Figure 2).

For several sources, as observed in similar investigations (e.g., Farrah et al. 2007), the estimated contributions to the MIR luminosity from star formation and an AGN do not add up to $100 \%$. In Figures 1 and 2, these sources are located either below or above the mixing line between the diagnostics, that is as the PAH EQW drops the $[\mathrm{Ne}$ V]/[Ne II] should go up, and galaxies should follow this line. However, many sources fall well below this mixing line. We suggest that this is partially due to uncertainties in the AGN zero points, that is the values of the

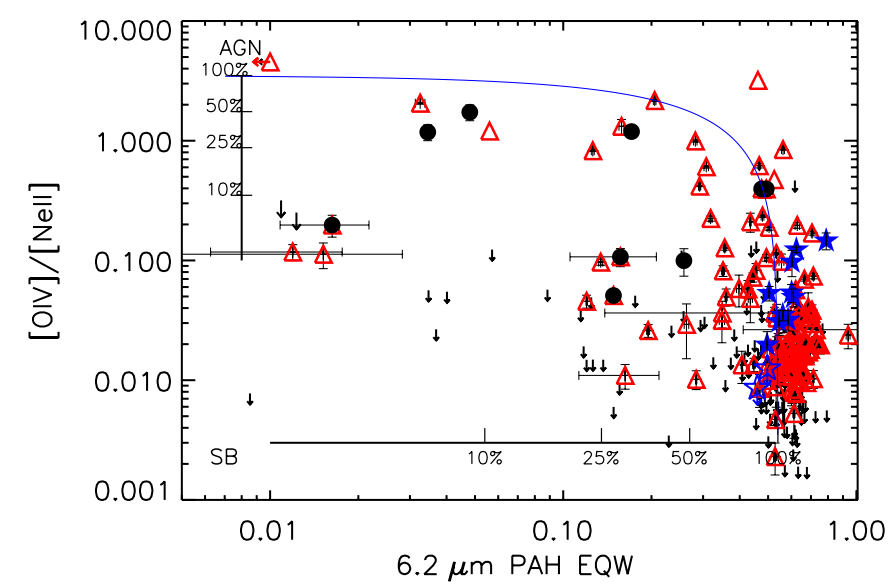

Figure 2. Mid-infrared [O IV]/[Ne II] vs. 6.2 $\mu \mathrm{m}$ PAH EQW excitation diagram. Symbols have the same definition as in Figure 1 except that red triangles indicate [O IV] detections.

(A color version of this figure is available in the online journal.)

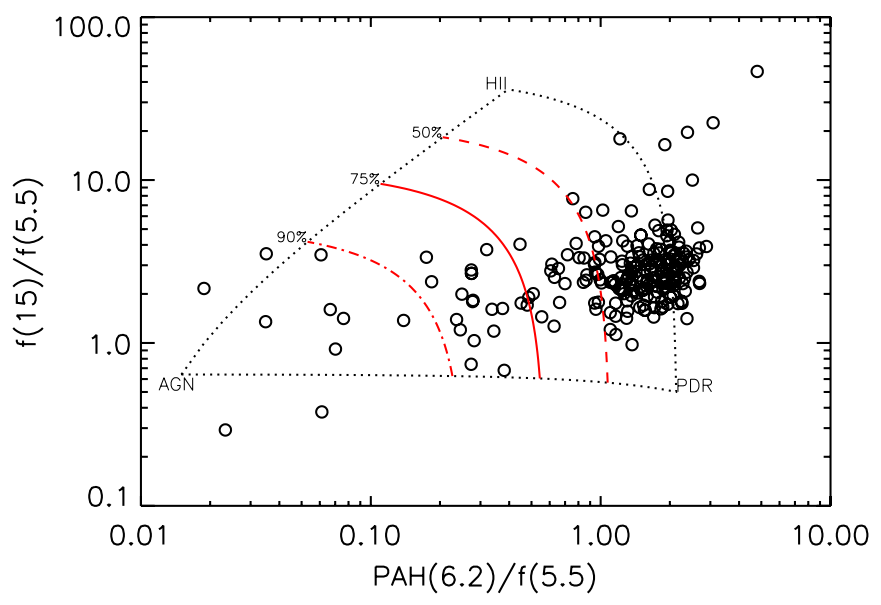

Figure 3. Mid-infrared diagnostic diagram, first used by Laurent et al. (2000) and later modified for Spitzer IRS by Armus et al. (2007), comparing the integrated continuum flux from 14 to $15 \mu \mathrm{m}$, the integrated continuum flux from 5.3 to 5.5, and the $6.2 \mu \mathrm{m}$ PAH flux. The three vertices, labeled as AGN, H II, and PDR, represent the positions of 3C273 from Weedman et al. (2005), and M17 and NGC 7023 from Peeters et al. (2004). These vertices were chosen to facilitate comparison with ULIRGs as presented in Armus et al. (2007) and Brandl et al. (2006). The red lines from left to right indicate a 90\%, 75\%, and 50\% fractional AGN contribution to the nuclear MIR luminosity, respectively. GOALS sources are shown as open circles.

(A color version of this figure is available in the online journal.)

$[\mathrm{Ne} \mathrm{v}] /[\mathrm{Ne} \mathrm{II}]$ and $[\mathrm{O} \mathrm{IV}] /[\mathrm{Ne}$ II] flux ratios for a source in which the AGN contributes $100 \%$ of the MIR luminosity. In addition differential extinction of the [Ne $\mathrm{V}]$ and [O IV] lines originating from the obscured region around the nucleus versus the [Ne II] line originating from the SB (likely more extended) may lead to an underestimation of the contribution of the AGN to the MIR emission.

\subsection{Dust Diagnostics}

For the sample of LIRGs in GOALS the $6.2 \mu \mathrm{m}$ PAH EQWs range between 0.01 and $0.94 \mu \mathrm{m}$ with a mean of 0.47 and a median of 0.53 . About $16 \%$ of LIRG nuclei have $6.2 \mu \mathrm{m}$ PAH EQW below $0.27 \mu \mathrm{m}$, less than one-half of the value seen in local SB systems (Brandl et al. 2006). This suggests that $16 \%$ of LIRGs have an AGN which dominates the MIR emission.

Figure 3 adopted from Laurent et al. (2000) and Armus et al. (2007) shows the $15-5.5 \mu \mathrm{m}$ continuum flux ratios versus the 6.2-5.5 $\mu \mathrm{m}$ continuum flux ratios for the LIRGs. The continuum 


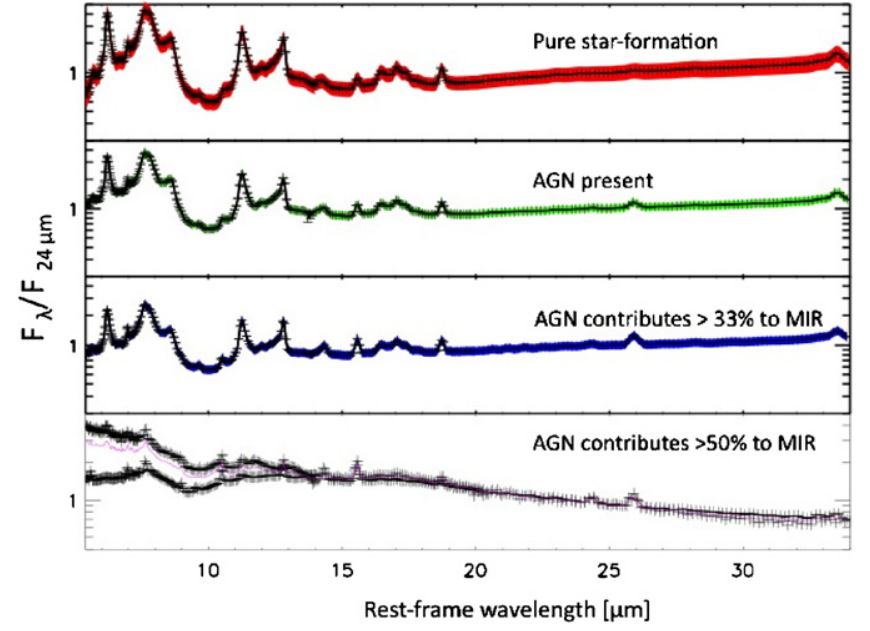

Figure 4. Average low-resolution spectra of four groups of galaxies. The spectra were normalized by the flux at $24 \mu \mathrm{m}$ and were weighted by the signal-to-noise ratio $(\mathrm{S} / \mathrm{N})$ at $24 \mu \mathrm{m}$. The error bars give the $1 \sigma$ error on the average while the shaded region shows the intrinsic weighted dispersion in the spectra that were combined to determine the average. From top to bottom we show averages of the following groups of objects. Group (1) contains sources without detectable $[\mathrm{Ne} \mathrm{v}]$ and with [O IV] $/[\mathrm{Ne}$ II] flux ratios $\leqslant 0.35$, that is sources whose MIR luminosity is dominated by star formation. Group (2) contains sources with $[\mathrm{Ne} \mathrm{v}]$ detections. Group (3) contains sources with $[\mathrm{Ne} \mathrm{v}] /[\mathrm{Ne}$ II $] \geqslant 0.14$ or $[\mathrm{O}$ IV $] /[\mathrm{Ne}$ II] flux ratios $\geqslant 0.5$ suggesting an AGN contribution to the MIR greater than $\sim 33 \%$. Group (4) contains galaxies with $[\mathrm{Ne} \mathrm{v]/[Ne} \mathrm{II]} \geqslant 0.75$ indicating an AGN contribution to the MIR greater than 50\%. Because we have usable SL spectra for only two out of the three galaxies in group (4), instead of showing the intrinsic weighted dispersion on the derived average spectra, we show the actual spectra of the two sources, normalized by the flux at $24 \mu \mathrm{m}$.

(A color version of this figure is available in the online journal.)

ratios measured in 3C273 (Weedman et al. 2005) were used to represent the expected values for a source in which the MIR luminosity comes only from an AGN, while those of M17 and NGC 7023 (Peeters et al. 2004) typify continuum ratios measured in pure $\mathrm{HII}$ regions and photo-dissociation regions (PDRs). The majority of the LIRGs nuclei fall in the PDR/H II region of the graph, $19 \%$ of objects have low $f_{15 \mu \mathrm{m}} / f_{5 \mu \mathrm{m}}$ and weak PAH emission suggesting an AGN contribution to the nuclear MIR emission greater than $50 \%$.

\subsection{Average Spectra}

In order to look for variations in the average spectra of LIRGs as a function of the relative importance of an AGN to the IR emission, we have combined low-resolution spectra, that did not have a large jump between SL and LL, spectra into groups based on detection of the [Ne v] $14.3 \mu \mathrm{m}$ emission line, and the strength of the $[\mathrm{Ne} \mathrm{V}] /[\mathrm{Ne}$ II] line flux ratio. Before they were combined, the spectra were normalized to the flux at $24 \mu \mathrm{m}$ and were weighted by the signal-to-noise ratio at $24 \mu \mathrm{m}$. The lowresolution spectra were divided into the following four groups. The first group contained sources without detectable [Ne v] and with $[\mathrm{O}$ IV $] /[\mathrm{Ne}$ II $]$ flux ratios $\leqslant 0.35$, that is sources whose nuclear MIR luminosity is dominated by star formation, there are 121 objects in this group. The second group contains sources with [ $\mathrm{Ne} \mathrm{v}]$ detections, there are 33 sources in this group. The third group contains all the sources with $[\mathrm{Ne} \mathrm{V}] /[\mathrm{Ne}$ II $] \geqslant 0.14$ or $[\mathrm{O}$ IV $] /[\mathrm{Ne}$ II $]$ flux ratios $\geqslant 0.5$ suggesting an AGN contribution to the MIR greater than $33 \%$ (five objects). The fourth group contains three sources with $[\mathrm{Ne} \mathrm{V}] /[\mathrm{Ne}$ II $] \geqslant 0.75$ indicating an AGN contribution to the nuclear MIR emission greater than $50 \%$. For one of the three sources in this group NGC 1068, we were not able to extract usable SL spectra because in the SL

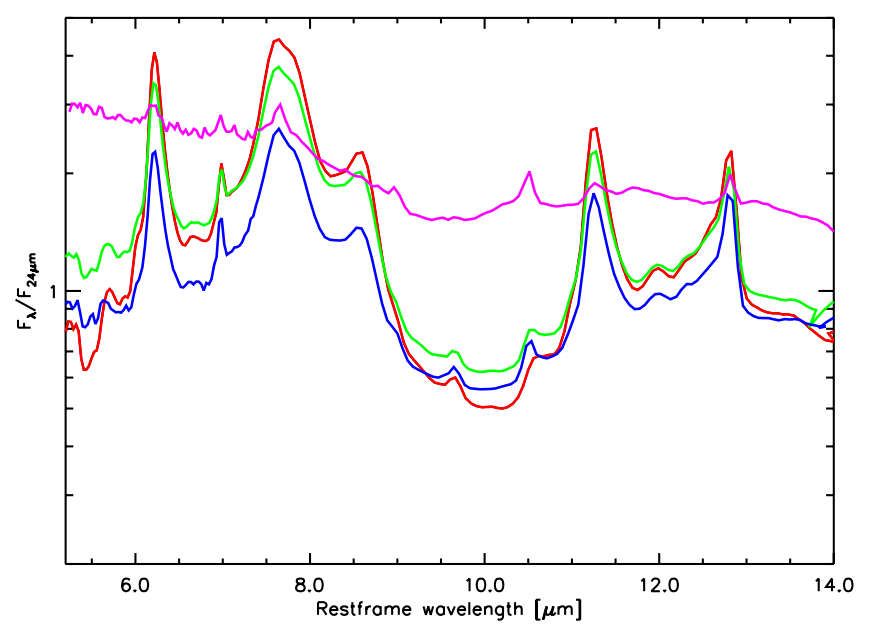

Figure 5. Average spectra of galaxies without detectable [Ne v] and with [O IV]/ [Ne II] flux ratios $\leqslant 0.35$ (red), with detectable [Ne v] emission (green), with $[\mathrm{Ne} \mathrm{v}] /[\mathrm{Ne}$ II $]$ flux ratios $\geqslant 0.14$ or $[\mathrm{O} \mathrm{IV}] /[\mathrm{Ne}$ II] flux ratios $\geqslant 0.5$ suggesting an AGN contribution to the MIR greater than $\sim 33 \%$ (blue), and the average spectra of two sources in which the AGN dominates the MIR emission (magenta).

(A color version of this figure is available in the online journal.)

map of this source the regions closest to the nucleus suffer from saturation (Howell et al. 2007). The spectra from each group were averaged and are shown in Figure 4.

Qualitatively the average spectra look similar, the broad spectral shapes of both are dominated by PAH emission and silicate absorption. The normalized and scaled comparison of the four average spectra are shown in Figure 5. Given the small number of sources where the $[\mathrm{Ne} v]$ emission lines indicate that the AGNs dominate the MIR emission, we only quantitatively compare groups (1) and (3). The PAH emission, as traced by the 6.2 and $11.3 \mu \mathrm{m}$ PAH EQWs is significantly stronger in the group (1) average (EQWs: $0.54 \mu \mathrm{m}$ and $0.55 \mu \mathrm{m}$ ) than in the average of group (3) objects (EQWs: 0.26 and $0.31 \mu \mathrm{m}$ ). Deeper ice and silicate absorption features are shown in the average spectra of the first group. A statistical analysis of the lowresolution spectra of all the GOALS sources will be presented in S. Stierwalt et al. (2011, in preparation).

The correspondence between spectral classification and the continuum slope in the mid-infrared has been discussed by a number of authors (e.g., Brandl et al. 2006; Veilleux et al. 2009a). The 30-15 $\mu \mathrm{m}$ flux ratio in particular has been used as a powerful diagnostic to separate the AGN from SB emission in high-redshift ULIRGs and QSOs (Veilleux et al. 2009a). The IR continuum slopes of the average spectra are compared by measuring the $30 \mu \mathrm{m}-15 \mu \mathrm{m}$ and $30 \mu \mathrm{m}-5.5 \mu \mathrm{m}$ continuum flux ratios. In the average spectra of group (1) sources, these continuum ratios are 1.6 and 1.8 , respectively, while in the average spectra of group (3) sources they both are 1.1, confirming the fact that more hot dust is present in sources with a significant $\geqslant 33 \%$ AGN contribution to the MIR. Therefore, the study presented here confirms that, on average, the $30 \mu \mathrm{m}-15 \mu \mathrm{m}$ and $30 \mu \mathrm{m}-5.5 \mu \mathrm{m}$ continuum flux ratios can also be used to estimate the relative AGN activity among LIRGs.

\section{DISCUSSION}

\subsection{AGN Contribution to the Total IR Luminosity}

In this section we compare the IRS AGN indicators and search for correlations between the AGN contribution to the IR and other properties such as the infrared colors, luminosities and merger stage. This paper presents several diagnostics that are 
effective at isolating the AGN contribution to the MIR emission in LIRG nuclei. The ratios of high-ionization [Ne V] and [O IV] line fluxes to the low-ionization [Ne II] line suggest that only $\sim 1 \%$ of LIRGs are AGN-dominated. The EQWs of the $6.2 \mu \mathrm{m}$ PAH feature indicate that $16 \%$ of sources are AGN-dominated. Diagnostics based on the shape of the MIR continuum imply that in $19 \%$ of the sources the AGN contributes more than $50 \%$ to the MIR luminosity.

Several factors may contribute to the apparent discrepancies between the diagnostics presented here. Among these are (1) ambiguities in the definition of the zero point (e.g., the value of the $[\mathrm{Ne} \mathrm{V}] /[\mathrm{Ne}$ II] and [O IV]/[Ne II] ratios for a pure AGN), (2) uncertainties in measuring the continuum under the $\mathrm{PAH}$ feature and in measuring the total feature's strength, (3) differences between the projected sizes of the wider $\mathrm{SH}$ and LH slits (used to measure the fine-structure lines) and the narrower SL slit (used to measure the $6.2 \mu \mathrm{m}$ PAH EQW), and (4) differential extinction between [ $\mathrm{NeV}],[\mathrm{O} I V]$, and [Ne II] that can affect the measured line ratios. Moreover, inherent in all the MIR diagnostics is the assumption that the bolometric correction is the same for all objects and that the lines accurately trace either the AGN or the star formation power. A number of authors (e.g., Armus et al. 2007; Veilleux et al. 2009a) have pointed out the failure of these assumptions among ULIRGs and this is expected to be true for LIRGs as well.

While the simple mixing lines of Figures 1-3 give an indication of the AGN contribution to the mid-infrared emission in LIRGs, the real goal is to estimate the fraction of the total bolometric luminosity contributed by an AGN and an SB in each source. To this end, bolometric corrections to the fine-structure lines [ $\mathrm{Ne} \mathrm{V}],\left[\mathrm{O}_{\mathrm{IV}}\right],[\mathrm{Ne} \mathrm{II}]$, and hot dust continuum at $\sim 6 \mu \mathrm{m}$ were applied as in Veilleux et al. (2009a). These authors use PG QSOs to estimate the expected $L_{[\mathrm{Nev}]} / L_{\mathrm{BOL}}, L_{\left[\mathrm{O}_{\text {Iv }}\right]} / L_{\mathrm{BOL}}$, and $L_{[\mathrm{Ne} \text { II] }} / L_{\mathrm{BOL}}$ for sources where the entire IR luminosity comes from an AGN. We estimate the [Ne II] to bolometric $\left(L_{[\mathrm{Ne} \text { II }} / L_{\mathrm{BOL}}\right)$ ratio for a source with no AGN contribution to the IR emission as the mean of the $L_{[\mathrm{Ne}}{ }_{\mathrm{II}} / L_{\mathrm{BOL}}$ ratio for all sources in this LIRG sample which (1) have $6.2 \mu \mathrm{m}$ PAH EQW $\geqslant 0.54 \mu \mathrm{m},(2)$ are not detected in [Ne v] nor [O IV], and (3) have $[\mathrm{Ne} \mathrm{V}] /[\mathrm{Ne} \mathrm{II}]$ and $[\mathrm{O} \mathrm{IV}] /[\mathrm{Ne} \mathrm{II}]$ upper limits of less than 0.1 and 1 , respectively. After applying those corrections we estimate the AGN contribution to the nuclear IR luminosity in each source. To determine the AGN contribution to the total IR luminosity we multiply these values by an aperture correction to account for MIR emission outside the slit. It is assumed that the MIR emission outside the slit is produced by young stars and spectral-mapping observations of LIRGs confirm this (e.g., Pereira-Santaella et al. 2010). This aperture correction is approximated from the ratio of nuclear to total MIPS $24 \mu \mathrm{m}$ flux. It is assumed that this ratio is the same as the ratio of nuclear to total MIR luminosity. At the median distance of the sample $(88 \mathrm{Mpc})$ the width of the SL slit projects to a size of $\sim 1.5 \mathrm{kpc}$. The ratio of $24 \mu \mathrm{m}$ flux measured with the IRS to the total measured with MIPS has a median of 0.93 and ranges from 0.27 to 1 . Thus, the average aperture corrections to the sample are small. This is especially true for the most luminous galaxies in the sample which are the most distant. After applying these adjustments we find that the whole set of diagnostics converges. We thus estimate that $9 \%-11 \%$ of LIRGs are AGN dominated (i.e., more than $50 \%$ of their IR luminosity is generated by an $\mathrm{AGN}$ ).

Since large samples of ULIRGs and low-luminosity galaxies have been observed with Spitzer, it is interesting to compare the published AGN fractions in those sources to those we have derived for local LIRGs. In ULIRGs $30 \%-40 \%$ of the sources are dominated by AGN dust heating (Armus et al. 2004, 2007; Desai et al. 2007; Farrah et al. 2007; Veilleux et al. 2009a). In local galaxies with $\left(L_{\mathrm{IR}} \leqslant 10^{11} L_{\odot}\right)$ this number is closer to $5 \%$ (Goulding \& Alexander 2009). The data discussed here suggest that LIRGs are powered mostly by star formation and that the AGN contribution to the IR is two times higher in LIRGs than it is in local normal galaxies but three to four times lower than in ULIRGs. The AGN contribution to the total IR luminosity is highest in the most luminous sources (e.g., Armus et al. 2007; Desai et al. 2007; Veilleux et al. 2009a; Goulding \& Alexander 2009).

Figure 6 which shows the IR luminosity versus the $6.2 \mu \mathrm{m}$ PAH EQW suggests that within the IR luminosity range of LIRGs there is no evidence of a linear trend between the AGN fraction and the IR luminosity. Extinction alone cannot explain the different detection statistics in LIRGS and ULIRGs. While the strength of the silicate absorption cannot be used to determine the differential extinction of [ $\mathrm{NeV}$ ] and [ $\mathrm{Ne}$ II] lines because of the necessary assumptions made about the dust geometry affecting these lines, in most LIRGs the silicate absorption is less deep than in ULIRGs (Pereira-Santaella et al. 2010; S. Stierwalt et al. 2011, in preparation). This suggests that ULIRGs may indeed have more extinction in the central few kiloparsecs than LIRGs and the lower AGN fractions among LIRGs cannot be due to extinction alone.

\subsection{Cumulative Contribution of $A G N$ to the IR Luminosity of the Sample}

In the previous section it has been estimated that about $10 \%$ of LIRGs are AGN-dominated. In this section the total contribution of AGN to the IR luminosity of the entire GOALS sample is computed.

The cumulative contribution of all AGNs to the total bolometric luminosity of the entire sample of local LIRGs was estimated as follows. ${ }^{20}$ (1) The $6.2 \mu \mathrm{m}$ PAH EQWs are used to estimate the nuclear AGN contribution to the total IR emission from each galaxy. (2) These values are then multiplied by an aperture correction to account for MIR emission outside the slit as described in the previous section. (3) The AGN IR luminosities for each galaxy are then summed and that sum is divided by the total IR luminosity of the sample. The resulting cumulative contribution of all AGNs in the sample to the total IR luminosity of the entire sample of LIRGs is $12 \%$.

\subsection{AGN Fractions as a Function of Luminosity and Color}

We find no obvious correlations between the total IR luminosity and the $[\mathrm{Ne} \mathrm{V}] /[\mathrm{Ne} \mathrm{II}]$ and $\left[\mathrm{O}_{\mathrm{IV}}\right] /[\mathrm{Ne} \mathrm{II}]$ emission line ratios (Figure 6).

Desai et al. (2007) find that the far-infrared spectral slope is correlated with the AGN contribution in ULIRGs and that despite a large scatter the $6.2 \mu \mathrm{m}$ PAH EQW decreases with increasing rest-frame $24 \mu \mathrm{m}$ luminosity and with increasing 25-60 IRAS flux ratios. Similar relations for LIRGs would permit determining the presence of AGN using only IR photometric studies.

Figures 7-9 show the distribution of total and nuclear $24 \mu \mathrm{m}$ luminosities and $f_{24} \mu \mathrm{m} / f_{70 \mu \mathrm{m}}$ flux ratios for the GOALS sample versus their $6.2 \mu \mathrm{m}$ PAH EQW. Figures 7-9 show very little

\footnotetext{
${ }^{20}$ As mentioned, we refer to the values measured within the IRS slit as nuclear values, and total or global values to those for the entire galaxy.
} 

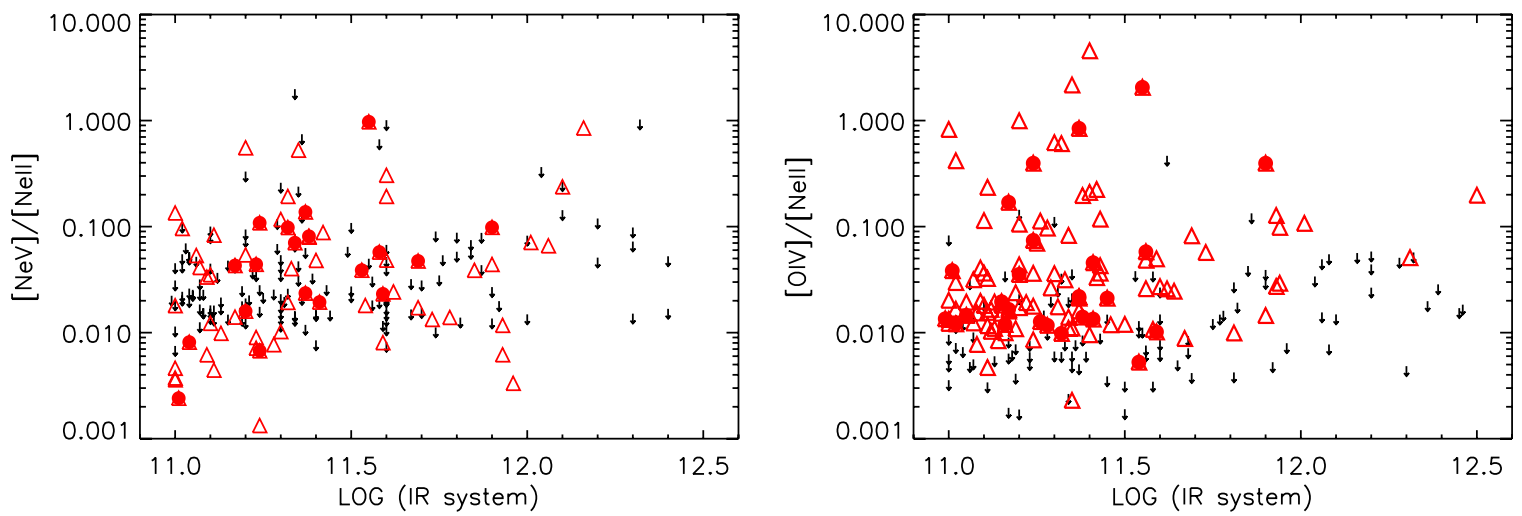

Figure 6. Strength of the [Ne v] feature (left) and [O Iv] (right) emission vs. the IR luminosity as estimated from MIPS fluxes (solid symbols) or from IRAS measurements (empty symbols).

(A color version of this figure is available in the online journal.)
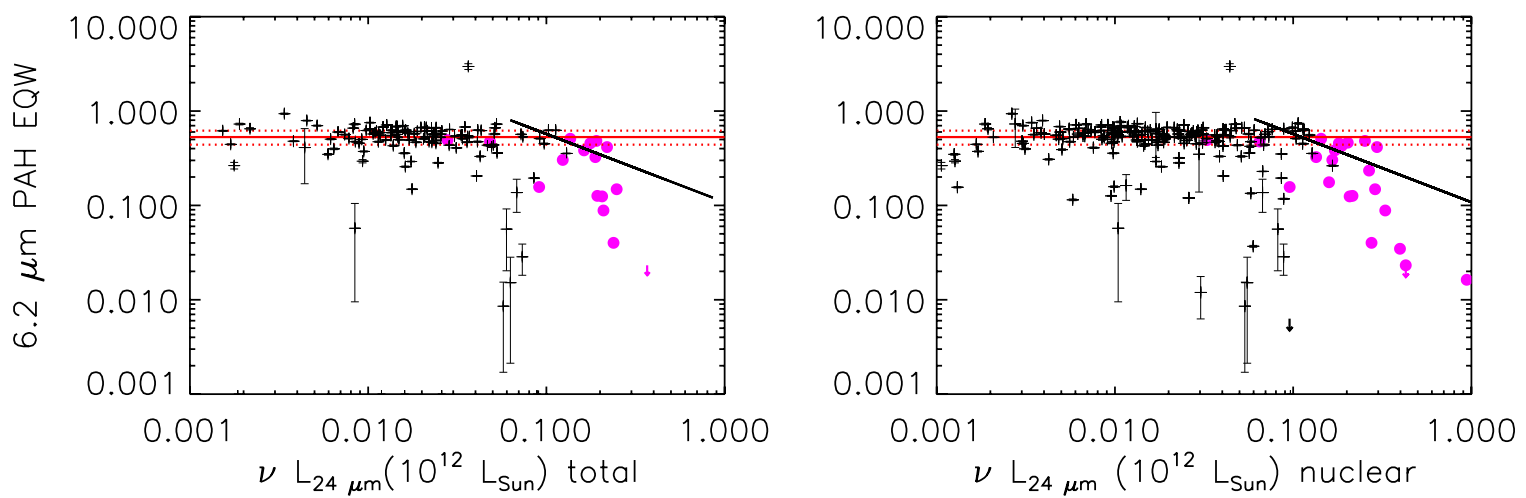

Figure 7. $6.2 \mu \mathrm{m}$ PAH EQW vs. $\log \left(24 \mu \mathrm{m}\right.$ luminosity) from MIPS $24 \mu \mathrm{m}$ estimates of the luminosity in an aperture of $1^{\prime}$ (left) and from the nuclear LL spectra with a slit width of 10'.7 (right). The 22 ULIRGs from the GOALS sample are shown in magenta filled circles, while the LIRGs are shown as black crosses. The red solid line marks where the $6.2 \mu \mathrm{m}$ PAH EQW equals $0.53 \mu \mathrm{m}$. This is the average EQW for SBs as determined by Brandl et al. (2006). The dotted red lines mark the $1 \sigma$ scatter in that value. While no obvious trend with luminosity can be distinguished, the median $6.2 \mu \mathrm{m}$ PAH EQW value for LIRGs is higher than that for ULIRGs. The black solid lines mark the relation between the $6.2 \mu \mathrm{m}$ PAH EQW and $\log (24 \mu \mathrm{m}$ luminosity) found by Desai et al. (2007).

(A color version of this figure is available in the online journal.)

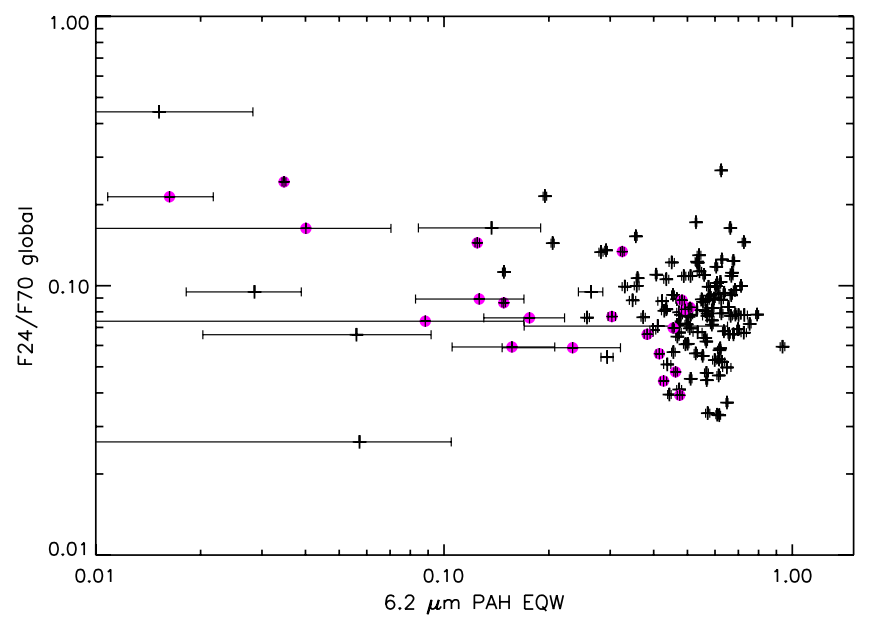

Figure 8. FIR colors vs. $6.2 \mu \mathrm{m}$ PAH EQW for sources where MIPS $24 \mu \mathrm{m}$ and MIPS $70 \mu \mathrm{m}$ nuclear fluxes were extracted. The ULIRGs from the GOALS sample are shown in magenta filled circles, while the LIRGs are shown in black crosses. No significant trend with IR colors can be distinguished, however the median MIPS $24 \mu \mathrm{m}$ and MIPS $70 \mu \mathrm{m}$ flux ratios for AGN-dominated sources are higher than those of star-formation-dominated sources.

(A color version of this figure is available in the online journal.)

scatter in the $6.2 \mu \mathrm{m}$ PAH EQW as a function of the total and nuclear $24 \mu \mathrm{m}$ luminosity in contrast to the 22 ULIRGs in the sample which shows a larger scatter. The median and mean values for $v L_{v}\left[10^{12} L_{\odot}\right]$ for sources with 6.2 PAH EQW $(\leqslant 0.27 \mu \mathrm{m})$ are 0.06 and 0.1 , respectively. The same quantities for sources with high $6.2 \mu \mathrm{m}$ PAH EQW $(\geqslant 0.54 \mu \mathrm{m})$ are 0.02 and 0.02. The range of values for the entire sample is 0.001-0.9.

Figure 7 also shows the distribution of nuclear $24 \mu \mathrm{m} \mathrm{lu}-$ minosities obtained from the LL spectra convolved with the response curve of MIPS $24 \mu \mathrm{m}$ filter and the results are similar. The mean values of $f_{24 \mu \mathrm{m} \text { MIPS }} / f_{70 \mu \mathrm{m} \text { MIPS }}$ flux ratios are 0.20 for sources with $6.2 \mu \mathrm{m}$ PAH $\leqslant 0.27 \mu \mathrm{m}$ EQW and 0.09 for sources with $6.2 \mu \mathrm{m}$ PAH EQW $\geqslant 0.54 \mu \mathrm{m}$. The range of values for the entire sample is $0.02-0.88$.

A combination of Spearman and Kolmogorov-Smirnov (K-S) tests suggests that local LIRGs with significant AGN contribution $(6.2 \mu \mathrm{m}$ PAH EQW $\leqslant 0.27 \mu \mathrm{m})$ have higher $L_{24} \mu \mathrm{m}$ and are slightly warmer than star-formation-dominated sources. The $\mathrm{K}-\mathrm{S}$ test indicates that the flux ratio distributions are different for these two groups of LIRGs with $98 \%$ significance. However, no tight correlation is found between the $6.2 \mu \mathrm{m}$ PAH EQW and the nuclear $f_{5 \mu \mathrm{m}} / f_{24 \mu \mathrm{m}}$ flux ratios (determined from the SL and LL spectra) or the global $24 \mu \mathrm{m}$ MIPS/70 $\mu \mathrm{m}$ MIPS flux ratios. It should be noted that Desai et al. (2007) used IRAS colors for the ULIRGs while the analysis presented here is done using MIPS fluxes determined using apertures of $1 \mathrm{arcmin}$ (J. M. Mazzarella et al. 2011, in preparation). However, in local LIRGs IRAS and MIPS fluxes are tightly proportional. The results presented here suggest that IR colors in LIRGs cannot 


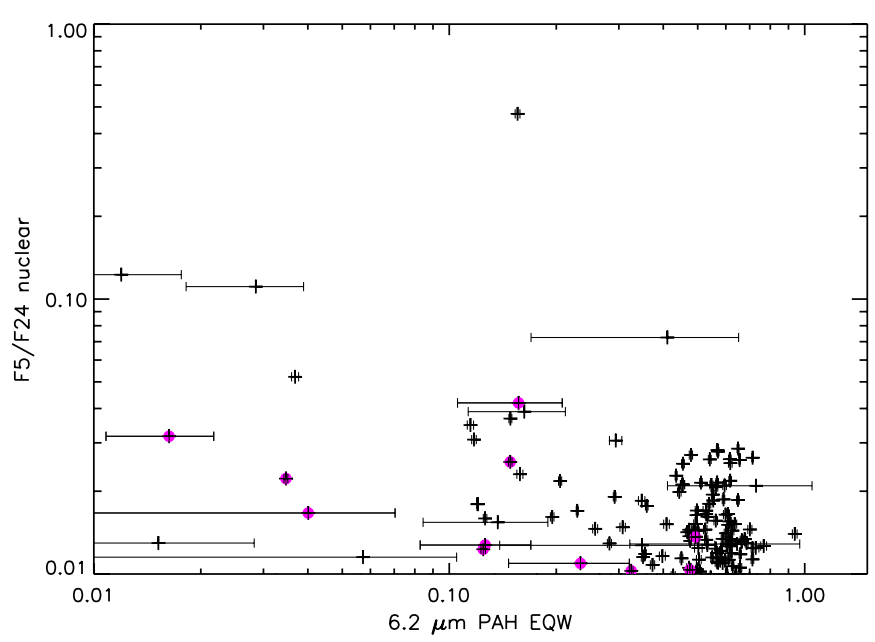

Figure 9. IR colors vs. $6.2 \mu \mathrm{m}$ PAH EQW. The $f_{5 \mu \mathrm{m}}$ and $f_{24} \mu \mathrm{m}$ nuclear fluxes were extracted from the low-resolution spectra. The ULIRGs from the GOALS sample are shown in magenta filled circles, while the LIRGs are shown in black crosses. No significant trend with IR colors can be distinguished, however the median $f_{5 \mu \mathrm{m}}-f_{24 \mu \mathrm{m}}$ flux ratios for AGN-dominated sources are higher than those of star-formation-dominated sources.

(A color version of this figure is available in the online journal.)

provide an exact estimate of the AGN contribution to the MIR emission in an individual LIRG.

\subsection{AGN Contribution versus Stage of Interaction}

The connections between galaxy growth through mergers and growth of the central supermassive black hole (SMBH) through accretion have been thoroughly studied theoretically and are continuously being investigated observationally. Most ULIRGs are advanced stage mergers (e.g., Murphy 2000; Surace 1998), while local LIRGs span a much wider range of interaction stages. For example, many of the lowest luminosity sources $\left(L_{\mathrm{IR}} \sim 10^{11} L_{\odot}\right)$ are single galaxies with only minor companions. Several studies of PG QSOs (e.g., Dasyra et al. 2006; Veilleux et al. 2009) and warm LIRGs (Yuan et al. 2010) have shown that AGN activity becomes increasingly dominant during the final merger stages. Here, we investigate whether this trend is evident among the large sample of local LIRGs in GOALS using MIR spectroscopic diagnostics to determine the presence of an AGN.

The GOALS sources were classified in five stages (see Figure 10 for examples): (0) no obvious sign of a disturbance either in the IRAC or HST morphologies, or published evidence that the gas is not in dynamical equilibrium (i.e., undisturbed circular orbits); (1) early stage, where the galaxies are within
1 arcmin of each other, but little or no morphological disturbance can be observed; (2) the galaxies exhibit bridges and tidal tails, but they do not have a common envelope and each optical disk is relatively intact; (3) the optical disks are completely destroyed but two nuclei can be distinguished; and (4) the two interacting nuclei are merged. The classification scheme was based on a combination of HST, IRAC 3.6, and DSS images. A search in the literature for detailed dynamical analysis based on H I data such as those in Yun et al. (2004) was also done. For the few sources with published $\mathrm{HI}$ interferometric data, the merger class was determined on the basis of that data. ${ }^{21}$ Note that this paper is not meant to be a comprehensive study of the merger properties of LIRGs. A more detailed study of the multi-wavelength morphologies of LIRGs as well as the effect of extinction on the merger classification will be presented elsewhere.

Figure 11 shows the histograms of merger stages for AGNdominated galaxies $(6.2 \mu \mathrm{m}$ EQW $\leqslant 0.27 \mu \mathrm{m})$ and non-AGN sources (sources without detectable [Ne v] emission and with $6.2 \mu \mathrm{m}$ EQW $\geqslant 0.53 \mu \mathrm{m})$ as well as the fraction of AGNdominated sources as a function of merger stage. We find no strong trends with merger stage. However, the fraction of AGNdominated sources is highest for galaxies in the latest merger stages, and the median $6.2 \mu \mathrm{m}$ EQW for non-merging galaxies is higher than that for merging systems. A K-S test indicates that the distribution of $6.2 \mu \mathrm{m}$ PAH EQWs of merging GOALS galaxies is different than that of non-merging sources at the $80 \%$ significance level. The last bin in particular is different at the $99 \%$ significance level when compared to the non-merger sources. This appears to be driven both by a decrease in the number of SBs and by an increase in the number of AGNdominated sources in the final stages of interaction. Sources with double nuclei in stages 1-3 are not significantly more AGN dominated than galaxies without obvious evidence of interaction. These results are consistent with models predicting that mergers of gas-rich spirals fuel both star formation and accretion onto an SMBH and that the AGNs become dominant in producing IR emission in the final mergers stages (e.g., Sanders et al. 1988; Veilleux et al. 2009). It is interesting to note that this increase in the AGN fraction in the late stage is driven by the ULIRG population. When the 22 GOALS ULIRGs are excluded from this analysis, the highest fractions of AGN-dominated sources are found in both early stage (1) and late stage (4) of interaction. The statistical significance of the difference between

\footnotetext{
21 For example, a source with an $\mathrm{H}_{\mathrm{I}}$ bridge was classified as stage 2 . $\mathrm{H}_{\mathrm{I}}$ observations are an established method of determining the interaction properties of galaxies and are thoroughly discussed in Hibbard \& van Gorkom (1996).
}
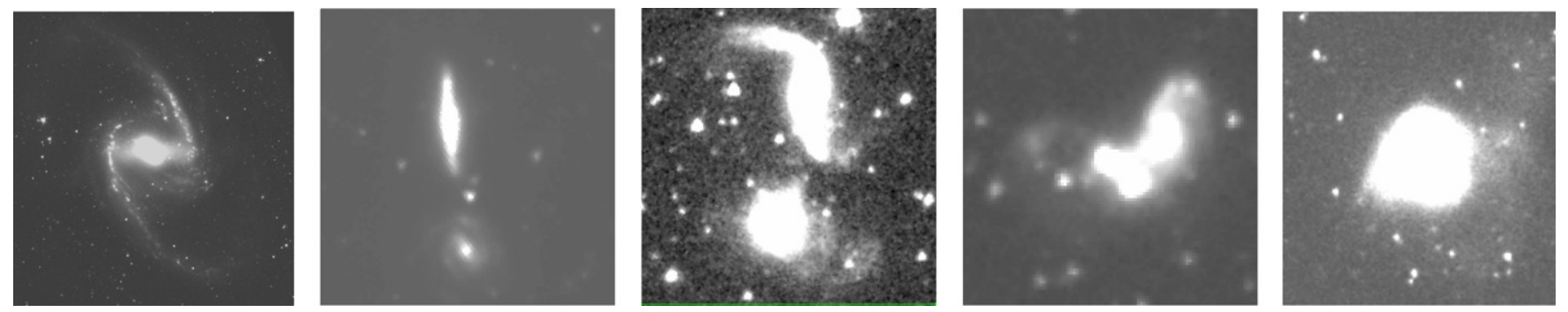

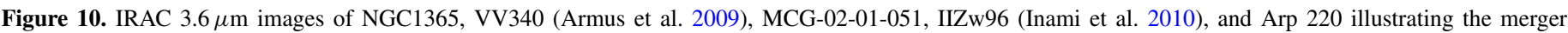

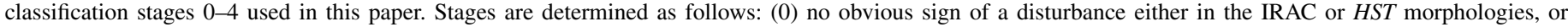

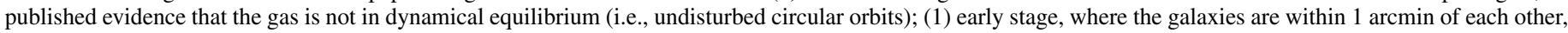

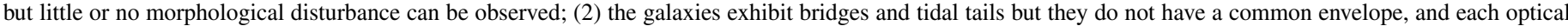

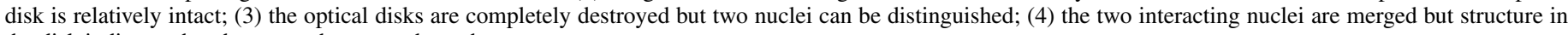
the disk indicates that the source has gone through a merger. 


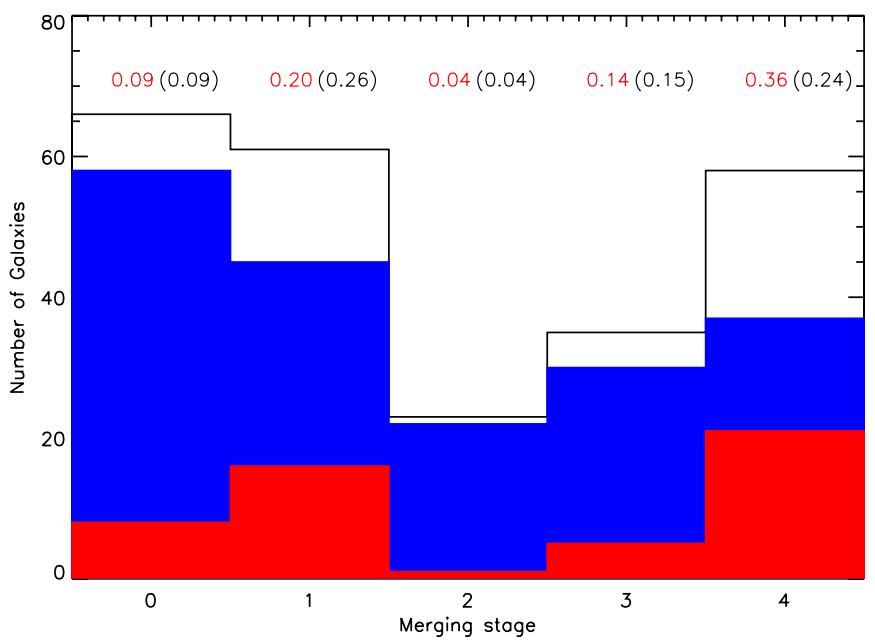

Figure 11. Distributions of AGN-dominated sources (i.e., $6.2 \mu \mathrm{m} \mathrm{PAH}$ EQW $\leqslant 0.27 \mu \mathrm{m}$ ) in red and SB objects (i.e., $6.2 \mu \mathrm{m}$ PAH EQW $\geqslant 0.53 \mu \mathrm{m}$ and without detectable [Ne v] emission) in blue as a function of merger stage (see Section 4.4). Atop each bin the fraction of AGN-dominated sources is written in red together with the same fraction obtained by excluding all ULIRGs (in black in parenthesis). The data is consistent with no trend between the number of AGN-dominated sources and the merger stage. However, the fraction of AGN-dominated sources is significantly higher (40\%) for sources in the last stage of merging. If ULIRGs are excluded from the analysis, the largest fractions (26\% and 24\%) of AGN-dominated LIRGs are found in the first and last merger stages, respectively.

(A color version of this figure is available in the online journal.)

mergers and non-mergers in the LIRG only sample decreases to $67 \%$ and the difference between the non-mergers and the last stage sources to $89 \%$. These results are consistent with the findings of Yuan et al. (2010) who studied a sample of optically classified LIRGs using optical spectroscopic classifications.

\subsection{IRAC Colors}

IR photometry in conjunction with other wavelengths has been used to significantly improve the methodology of finding obscured AGNs (e.g., Barkhouse \& Hall 2001; Glikman et al. 2004; Lacy et al. 2004; Stern et al. 2005). AGNs exhibit a pseudo power-law continuum in the MIR from dust grains heated to a wide range of temperatures above $100 \mathrm{~K}$ (e.g., Marshall et al. 2007). The SEDs arising from each of three distinct sources (AGN, H II region, and PDR) which heat the dust are sufficiently different to allow for an AGN selection based on their colors in the range of 4-24 $\mu \mathrm{m}$ (e.g., Sajina et al. 2005; Laurent et al. 2000; Lacy et al. 2007; Glikman et al. 2004; Richards et al. 2006; Stern et al. 2005; Hatziminaoglou et al. 2005).

Figure 12 shows the IRAC colors for the GOALS sample of LIRGs. All LIRGs chosen to have AGN-like IRAC colors (Stern et al. 2005) also have $6.2 \mu \mathrm{m}$ PAH EQW $\leqslant 0.27 \mu \mathrm{m}$ indicating a contribution upward of $\sim 50 \%$ to the IR luminosity. However, about half of all sources with low $6.2 \mu \mathrm{m}$ PAH EQW fall outside the AGN selection wedge. The sources with low $6.2 \mu \mathrm{m}$ PAH EQW outside the edge tend to be weaker AGNs as evidenced by (1) only one of those has a weak [Ne v] line and (2) their $\left[\mathrm{O}_{\mathrm{IV}}\right] /[\mathrm{Ne} \mathrm{II}]$ ratios are below 0.2. This suggests that the IRAC color-color method is effective at selecting LIRGs with powerful AGNs, but may fail toward the fainter end for LIRGs with an MIR luminosity arising mostly from star formation. The Lacy et al. (2004) IRAC color-color method selects $64 \%$ of the AGN-dominated LIRGs. However, SB galaxies are also selected as AGN, representing $43 \%$ of all the sources selected with this method. Therefore, for our sample of LIRGs this method finds

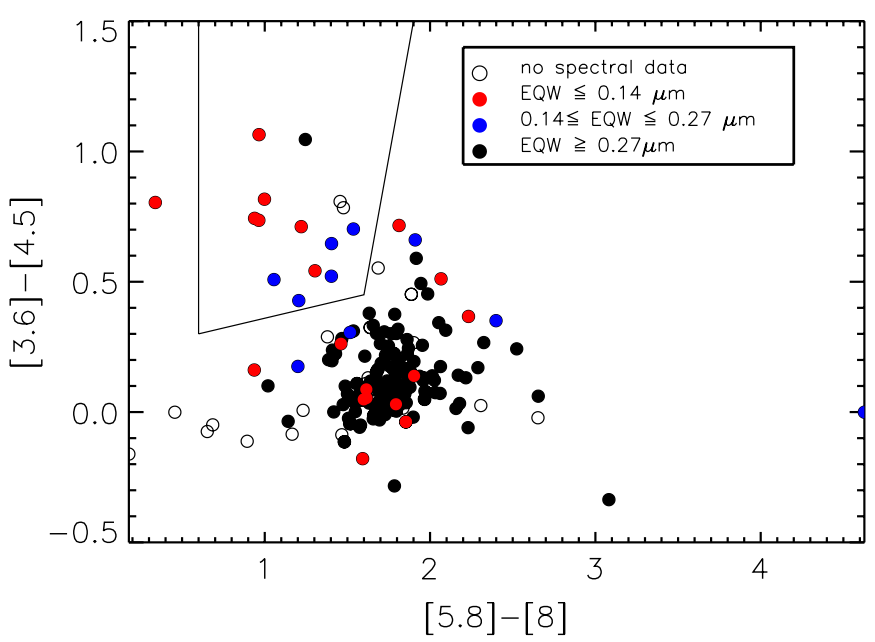

Figure 12. IRAC color $([5.8 \mu \mathrm{m}]-[8 \mu \mathrm{m}])$ and $([3.6 \mu \mathrm{m}]-[4.5 \mu \mathrm{m}])$ plot of 224 LIRG nuclei. The IRAC total fluxes shown here are from apertures matched to the MIPS $24 \mu \mathrm{m}$ apertures of typically $1^{\prime}$, but range between 0.5 and $1^{\prime} .5(\mathrm{~J}$. M. Mazzarella et al. 2011, in preparation). The solid lines show the color cuts which Stern et al. (2005) use to separate active galaxies from Galactic stars and normal galaxies. The solid black, red, and blue dots represent points with $6.2 \mu \mathrm{m}$ PAH EQW greater than $0.27 \mu \mathrm{m}$, between 0.14 and $0.27 \mu \mathrm{m}$ and smaller than $0.14 \mu \mathrm{m}$, respectively. The empty circles represent nuclei without MIR spectra. (A color version of this figure is available in the online journal.)

more AGNs but also selects objects that have their IR emission produced by dust heated only by star formation.

\section{CONCLUSIONS}

This paper presents a statistical analysis of 248 LIRG spectra in the rest-frame wavelength range between 5 and $38 \mu \mathrm{m}$. Several diagnostics effective at isolating the AGN contribution to the MIR emission using [Ne v], [O IV], and [Ne II] gas lines, the $6.2 \mu \mathrm{m}$ PAH EQW, and the shape of the MIR continuum are compared. The summary is as follows.

1. The high-ionization emission lines of [Ne v] $14.322 \mu \mathrm{m}$ and [O IV] $25.890 \mu \mathrm{m}$ are detected in $18 \%$ and $53 \%$ of all LIRG nuclei, respectively. Since the [Ne v] line does not arise from gas heated by hot stars, its detections suggest the presence of an AGN in at least $18 \%$ of LIRG nuclei.

2. Diagnostics using the $[\mathrm{Ne} \mathrm{v}] /[\mathrm{Ne}$ II $]$, [O IV]/[Ne II] line flux ratios, the $6.2 \mu \mathrm{m}$ PAHEQW, and the MIR continuum shape suggest that in $10 \%$ of local LIRGs the AGN dominates the bolometric luminosity. The vast majority of LIRGs are SB dominated. The fraction of local LIRG IR emission coming from an AGN as estimated in the mid-infrared is approximately two times larger than that seen in normal galaxies $(\sim 5 \%)$ and about three to four times lower than that seen in ULIRGs alone.

3. Summing the bolometric luminosity contributed by each AGN in the sample and dividing by the total IR luminosity of all the LIRGs suggests that AGNs are responsible for $\sim 12 \%$ of the total bolometric luminosity of local LIRGs.

4. In LIRGs there are no strong correlations between the fraction of IR luminosity from an AGN and the total or nuclear $24 \mu \mathrm{m}$ luminosity, the $24-60 \mu \mathrm{m}$ flux ratios or the interaction stage of the system. However, AGN-dominated LIRGs tend to be more luminous at $24 \mu \mathrm{m}$ and to have warmer IR colors than SB-dominated LIRGs.

5. By separating the GOALS sources according to merger stage it is found that there is a significant increase in the fraction of AGN-dominated sources among those galaxies 
in the latest stages of interaction. This trend is driven by the ULIRGs in the sample, since these objects tend to be late stage mergers and have larger AGN fractions than the LIRGs. This is consistent with findings of previous authors using optical diagnostics for LIRGs, MIR studies of ULIRGs and PG QSOs, and with models which predict that mergers of gas-rich spirals fuel both star formation and accretion onto an SMBH.

6. An investigation of the IRAC colors (i.e., [3.6 $\mu \mathrm{m}]-$ $[4.5 \mu \mathrm{m}]$ versus $[5.8 \mu \mathrm{m}]-[8 \mu \mathrm{m}])$, as introduced in Stern et al. (2005), of LIRGs indicates that only $50 \%$ of objects with a significant AGN contribution to the MIR emission fall within the range typically associated with AGN. The Lacy et al. (2004) AGN IRAC color criteria select a slightly higher fraction of the AGN-dominated LIRGs (64\%), at the expense of also including 11 LIRGs that appear SB dominated from their IRS spectra.

The measurements we present in this paper provide an estimate of the fraction of IR emission in LIRGs coming from AGNs. Our results provide an important local benchmark to be compared to high-redshift samples of LIRGs, especially those at epochs where the contribution of LIRGs to the IR background (e.g., at $z \sim 1$; see Magnelli et al. 2009) becomes substantial.

These diagnostics probe the nuclear source of IR emission in those LIRGs. A full understanding of the processes leading to the generation of LIRG activity requires careful analysis of the mass, temperature, and kinematics of the gas fueling and being heated by the star formation and AGN activity in LIRGs. Future papers (A. O. Petric et al. 2011, in preparation) will discuss the observations of warm and cold molecular gas in the GOALS sample and relate these to the energy sources and evolutionary state of the LIRGs.

We thank the anonymous referee for his comments which have significantly improved our paper. V.C. acknowledges partial support from the EU grants ToK 39965 and FP7REGPOT 206469. A.P. thanks N. Flagey for multiple readings of the document and comments which helped improve the clarity of this text. A.P. also thanks V. Desai for help with the data analysis, and $\mathrm{C}$. Bridge for discussions and help with the merger classification of the LIRGs in this paper.

This work is based primarily on observations made with the Spitzer Space Telescope, which is operated by the Jet Propulsion Laboratory, California Institute of Technology under NASA contract 1407.

We have made use of the NASA/IPAC Extra-galactic Database (NED) which is operated by the Jet Propulsion Laboratory, California Institute of Technology, under contract with NASA. Support for this research was provided by NASA through an award issued by JPL/Caltech.

\section{REFERENCES}

Alonso-Herrero, A., Pereira-Santaella, M., Rieke, G. H., Colina, L., Engelbracht, C. W., Pérez-González, P. G., Díaz-Santos, T., \& Smith, J.-D. T. 2010, Adv. Space Res., 45, 99

Alonso-Herrero, A., Quillen, A. C., Simpson, C., Efstathiou, A., \& Ward, M. J. 2001, AJ, 121, 1369

Armus, L., et al. 2004, ApJS, 154, 178

Armus, L., et al. 2006, ApJ, 640, 204

Armus, L., et al. 2007, ApJ, 656, 148

Armus, L., et al. 2009, PASP, 121, 559

Barkhouse, W. A., \& Hall, P. B. 2001, AJ, 121, 2843

Bernard-Salas, J., et al. 2009, ApJS, 184, 230
Brandl, B. R., et al. 2006, ApJ, 653, 1129

Caputi, K. I., et al. 2006, A\&A, 454, 143

Chary, R., \& Elbaz, D. 2001, ApJ, 556, 562

Chary, R., et al. 2004, ApJS, 154, 80

Dale, D. A., et al. 2006, ApJ, 646, 161

Dasyra, K. M., et al. 2006, New Astron. Rev., 50, 720

Desai, V., et al. 2007, ApJ, 669, 810

Díaz-Santos, T., et al. 2010, ApJ, 723, 993

Dudik, R. P., Weingartner, J. C., Satyapal, S., Fischer, J., Dudley, C. C., \& O'Halloran, B. 2007, ApJ, 664, 71

Elbaz, D., et al. 2002, A\&A, 384, 848

Evans, A. S., et al. 2008, ApJ, 675, L69

Farrah, D., et al. 2007, ApJ, 667, 149

Flores, H., et al. 1999, A\&A, 343, 389

Franceschini, A., Aussel, H., Cesarsky, C. J., Elbaz, D., \& Fadda, D. 2001, A\&A, 378,1

Genzel, R., \& Cesarsky, C. J. 2000, ARA\&A, 38, 761

Genzel, R., et al. 1998, ApJ, 498, 579

Gispert, R., Lagache, G., \& Puget, J. L. 2000, A\&A, 360, 1

Glikman, E., et al. 2004, ApJ, 607, 60

Gorjian, V., Cleary, K., Werner, M. W., \& Lawrence, C. R. 2007, ApJ, 655, L73

Goulding, A. D., \& Alexander, D. M. 2009, MNRAS, 398, 1165

Granato, G. L., Danese, L., \& Franceschini, A. 1997, ApJ, 486, 147

Hatziminaoglou, E., et al. 2005, AJ, 129, 1198

Hibbard, J. E., \& van Gorkom, J. H. 1996, AJ, 111, 655

Howell, J. H., et al. 2007, AJ, 134, 2086

Howell, J. H., et al. 2010, ApJ, 715, 572

Inami, H., et al. 2010, AJ, 140, 63

Iwasawa, K., et al. 2011, arXiv:1101.3659

Kim, D.-C., Veilleux, S., \& Sanders, D. B. 1998, ApJ, 508, 627

Lacy, M., et al. 2004, ApJS, 154, 166

Lacy, M., et al. 2007, ApJ, 669, L61

Lagache, G., Dole, H., \& Puget, J. 2003, MNRAS, 338, 555

Laurent, O., et al. 2000, A\&A, 359, 887

Le Floc'h, E., et al. 2005, ApJ, 632, 169

Levenson, N. A., Elitzur, M., Geballe, T. R., Mason, R. E., Nenkova, M., \& Sirocky, M. M. 2007, RevMexAA Conf. Ser., 29, 159

Lutz, D., Veilleux, S., \& Genzel, R. 1999, ApJ, 517, L13

Magnelli, B., et al. 2009, A\&A, 496, 57

Marleau, F. R., et al. 2004, ApJS, 154, 66

Marshall, J. A., et al. 2007, ApJ, 670, 129

Murphy, T. W., Jr. 2000, PhD thesis, California Institute of Technology

Nenkova, M., Ivezić, Ž., \& Elitzur, M. 2002, ApJ, 570, L9

Peeters, E., Spoon, H. W. W., \& Tielens, A. G. G. M. 2004, ApJ, 613, 986

Pereira-Santaella, M., Alonso-Herrero, A., Rieke, G. H., Colina, L., DíazSantos, T., Smith, J.-D. T., Pérez-González, P. G., \& Engelbracht, C. W. 2010, ApJS, 188, 447

Pier, E. A., \& Krolik, J. H. 1992, ApJ, 399, L23

Richards, G. T., et al. 2006, ApJS, 166, 470

Rigopoulou, D., et al. 1999, AJ, 118, 2625

Sajina, A., Lacy, M., \& Scott, D. 2005, ApJ, 621, 256

Sanders, D. B., Mazzarella, J. M., Kim, D., Surace, J. A., \& Soifer, B. T. 2003, AJ, 126, 1607

Sanders, D. B., \& Mirabel, I. F. 1996, ARA\&A, 34, 749

Sanders, D. B., Soifer, B. T., Elias, J. H., Neugebauer, G., \& Matthews, K. 1988, ApJ, 328, L35

Smith, J. D. T., et al. 2007, PASP, 119, 1133

Spoon, H. W. W., et al. 2007, ApJ, 654, L49

Stanford, S. A., Stern, D., van Breugel, W., \& De Breuck, C. 2000, ApJS, 131, 18

Stern, D., et al. 2005, ApJ, 631, 163

Strauss, M. A., Huchra, J. P., Davis, M., Yahit, A., Fisher, K. B., \& Tonry, J. 1992, ApJS, 83, 29

Sturm, E., et al. 2000, A\&A, 358, 481

Sturm, E., et al. 2002, A\&A, 393, 821

Surace, J. A. 1998, PhD thesis, Univ. Hawaii

Tran, Q. D., et al. 2001, ApJ, 552, 527

Veilleux, S., et al. 2009, ApJ, 701, 587

Veilleux, S., et al. 2009a, ApJS, 182, 628

Verma, A., et al. 2003, A\&A, 403, 829

Weedman, D. W., et al. 2005, ApJ, 633, 706

Wu, Y., Charmandaris, V., Huang, J., Spinoglio, L., \& Tommasin, S. 2009, ApJ, 701,658

Yuan, T., Kewley, L. J., \& Sanders, D. B. 2010, ApJ, 709, 884

Yun, M. S., Reddy, N. A., Scoville, N. Z., Frayer, D. T., Robson, E. I., \& Tilanus, R. P. J. 2004, ApJ, 601, 723 